\title{
Surface instability in nodal noncentrosymmetric superconductors
}

\author{
Carsten Timm,, 用 Stefan Rex, ${ }^{2}$ and P. M. R. Brydon ${ }^{3, \dagger}$ \\ ${ }^{1}$ Institute of Theoretical Physics, Technische Universität Dresden, 01062 Dresden, Germany \\ ${ }^{2}$ Department of Physics, Norwegian University of Science and Technology, 7491 Trondheim, Norway \\ ${ }^{3}$ Condensed Matter Theory Center and Joint Quantum Institute, \\ University of Maryland, College Park, USA 20742
}

(Dated: April 8, 2015)

\begin{abstract}
We study the stability of topologically protected zero-energy flat bands at the surface of nodal noncentrosymmetric superconductors, accounting for the alteration of the gap near the surface. Within a selfconsistent mean-field theory, we show that the flat bands survive in a broad temperature range below the bulk transition temperature. There is a second transition at a lower temperature, however, below which the system spontaneously breaks time-reversal symmetry. The surface bands are shifted away from zero energy and become weakly dispersive. Simultaneously, a spin polarization and an equilibrium charge current develop in the surface region.

PACS numbers: $\quad$ 74.20.Rp, 73.20.At, 74.25.Ha, 74.25.Jb
\end{abstract}

Introduction. The topological properties of gapless electronic systems have recently attracted much attention [1] 6]. An important example are time-reversalsymmetric noncentrosymmetric superconductors (NCSs) [1, 2, 7-11, which are characterized by strong antisymmetric spin-orbit coupling (SOC) and a parity-mixed pairing state 12]. Many NCSs display evidence of gaps with line nodes [13 18. This is exciting, as the line nodes of NCSs with dominant triplet pairing are topologically nontrivial defects in momentum space [1]. Zero-energy flat bands of Majorana fermions are predicted to appear within the projections of these nodal lines onto the surface Brillouin zone (BZ). Such flat bands have clear experimental signatures such as sharp zero-bias peaks in tunneling spectra [2, 11, equilibrium currents parallel to the interface between the NCS and a ferromagnet [19, 20, and characteristic quasiparticle interference patterns [21].

The topological properties of NCSs and consequently the protection of the surface states are controlled by the superconducting gaps, which arise from interactions. Properly accounting for these interactions may qualitatively alter the surface physics. For example, a surface tends to suppress some gap components and enhance others 22 28]. This may change the conclusions of the aforementioned studies [1-6, 911, which imposed unrealistic uniform gaps. Flat bands with their high density of states are particularly prone to instabilities. Indeed, the zero-energy flat bands at the (110) surface of $d$-wave superconductors with time-reversal symmetry (TRS) 29] are predicted to be unstable towards a time-reversalsymmetry-breaking (TRSB) state 22, 28, 30. This has been supported by some tunneling and transport experiments 3133 but was not seen in others 3438 . $d$-wave superconductors are however qualitatively different from NCSs in that the zero-energy flat bands are degenerate in the first case but nondegenerate in the second.

\footnotetext{
* carsten.timm@tu-dresden.de

$\dagger$ pbrydon@umd.edu
}

In this paper, we study the stability of the surface zeroenergy flat bands of nodal NCSs by performing selfconsistent mean-field (MF) calculations in real space for a slab of finite thickness. For concreteness, we consider a model with point group $C_{4 v}$, which is realized for $\mathrm{CePt}_{3} \mathrm{Si}$ [39, $\mathrm{CeRhSi}_{3}$ [40, and $\mathrm{CeIrSi}_{3}$ [1]. We show that an instability to a TRSB state can occur and study its signatures.

Model and mean-field theory. We start from a tightbinding Hamiltonian for an NCS with $C_{4}$ point group, $H=H_{0}+H_{\text {int }}$. The noninteracting part is

$$
\begin{aligned}
H_{0}= & -\mu \sum_{j} c_{j}^{\dagger} c_{j}-t \sum_{\langle i j\rangle}\left(c_{i}^{\dagger} c_{j}+c_{j}^{\dagger} c_{i}\right) \\
& +i \lambda \sum_{\langle i j\rangle}\left(\hat{\mathbf{z}} \times \hat{\mathbf{e}}_{i j}\right) \cdot\left(c_{i}^{\dagger} \frac{\boldsymbol{\sigma}}{2} c_{j}-c_{j}^{\dagger} \frac{\boldsymbol{\sigma}}{2} c_{i}\right),
\end{aligned}
$$

with the chemical potential $\mu$, the nearest-neighbor hopping amplitude $t$, and the Rashba SOC strength $\lambda$. The SOC term breaks inversion symmetry. The annihilation operator $c_{j}=\left(c_{j, \uparrow}, c_{j, \downarrow}\right)^{T}$ is a two-component spinor, $\boldsymbol{\sigma}$ is the vector of Pauli matrices, and $\hat{\mathbf{e}}_{i j}$ is the unit vector pointing from site $j$ to site $i$ of a simple cubic lattice. Attractive interactions at the same site and between nearest neighbors in the $x y$ plane are described by

$$
H_{\mathrm{int}}=-U_{s} \sum_{j} c_{j \uparrow}^{\dagger} c_{j \downarrow}^{\dagger} c_{j \downarrow} c_{j \uparrow}-U_{t} \sum_{\langle i j\rangle \perp \hat{\mathbf{z}} \sigma \sigma^{\prime}} c_{i \sigma}^{\dagger} c_{j \sigma^{\prime}}^{\dagger} c_{j \sigma^{\prime}} c_{i \sigma} .
$$

The interaction is decoupled in the pairing channel. We define the singlet and triplet order parameters $\Delta_{j}^{s} \equiv$ $\left(U_{s} / 2\right)\left\langle c_{j}^{T} i \sigma^{y} c_{j}\right\rangle$ and $\boldsymbol{\Delta}_{i j}^{t} \equiv i U_{t}\left\langle c_{j}^{T} i \sigma^{y} \boldsymbol{\sigma} c_{i}\right\rangle$, respectively, where the site indices $i, j$ in $\boldsymbol{\Delta}_{i j}^{t}$ are restricted to nearestneighbor sites in the $x y$ plane. The triplet vector order parameter is taken to be parallel to the effective SOC field, $\boldsymbol{\Delta}_{i j}^{t}=\Delta_{i j}^{t} \hat{\mathbf{z}} \times \hat{\mathbf{e}}_{i j}$. This choice avoids the tripletpair-breaking effect of the SOC, and is therefore energetically favorable in the bulk [42.

We first consider the MF solution for an extended system, assuming spatially uniform gaps $\Delta_{j}^{s}=\Delta_{s}$ and $\Delta_{i j}^{t}=\Delta_{t}$. Details of the calculation are given in Sec. I of 

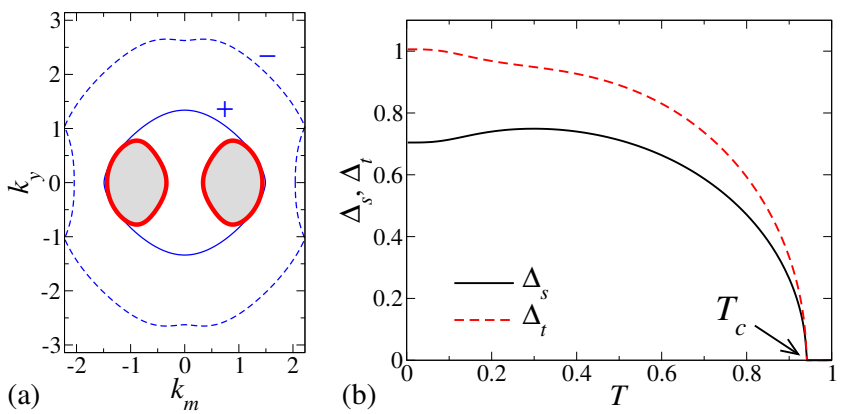

FIG. 1. (Color online) (a) Projection onto the (101) plane of the positive-helicity Fermi surface (thin solid line), the negative-helicity Fermi surface (dashed line), and the superconducting gap nodes on the former (heavy solid lines), for the bulk NCS. The gray areas denote the zero-energy flat bands predicted to exist at (101) surfaces under the assumption of uniform gaps [1, 2]. The plot is restricted to momenta in the (101) surface BZ, where $k_{m}=\left(k_{x}-k_{z}\right) / \sqrt{2}$. The parameters are $t=1, \lambda=1.5, \mu=-3, U_{s}=5.0, U_{t}=5.4$, and $T=0.0025$. (b) Mean-field gaps $\Delta_{s}$ (solid black) and $\Delta_{t}$ (dashed red) as functions of temperature $T$.

the Supplemental Material [4]. We find that the singlet and triplet gaps have the same phase, which can be set to zero, so that TRS is preserved. SOC splits the bands and thus also the Fermi surface according to the helicity of states 2. Since the triplet order parameter is parallel to the SOC, pairing only occurs between states with the same helicity. We determine interaction strengths $U_{s}, U_{t}$ that lead to flat zero-energy surface bands under the assumption of uniform gaps. The resulting surface states have been studied in detail in Refs. [2, 11, 44]. This is realized for the parameters $t=1$ (hence, $t$ is our unit of energy), $\lambda=1.5, \mu=-3, U_{s}=5.0, U_{t}=5.4$ at the temperature $T=0.0025$ (setting $k_{B}=1$ ), giving bulk MF gaps $\Delta_{s}=0.704$ and $\Delta_{t}=1.006$. We consequently find a gap with line nodes on the (smaller) positive-helicity Fermi surface, but a full gap on the (larger) negativehelicity Fermi surface 45. Figure 1(a) shows the projection of the two Fermi surfaces and the nodal lines onto the (101) plane. The topological argument from Refs. 1, 2 predicts that a (101) surface hosts flat zero-energy bands within the region bounded by the projected nodal lines. In addition, there is an arc of zero-energy states connecting the two regions with flat bands 2,5 , 11. Figure 1 (b) shows the bulk gaps $\Delta_{s}$ and $\Delta_{t}$ as functions of temperature.

We next turn to the MF solution for a slab of thickness $W$ with (101) surfaces. We introduce new coordinates $x=m+(l+l \bmod 2) / 2$ and $z=-m+(l-l \bmod 2) / 2$, where $m$ is parallel to the surfaces and $l=0, \ldots, W-1$ is orthogonal to them. The geometry of one surface and our coordinate system are depicted in the inset of Fig. 2. Since translational symmetry in the normal direction is broken, the gaps depend on $l$. We define

$$
\frac{U_{s}}{2}\left\langle c_{j}^{T} i \sigma^{y} c_{j}\right\rangle \equiv \Delta_{l}^{s},
$$

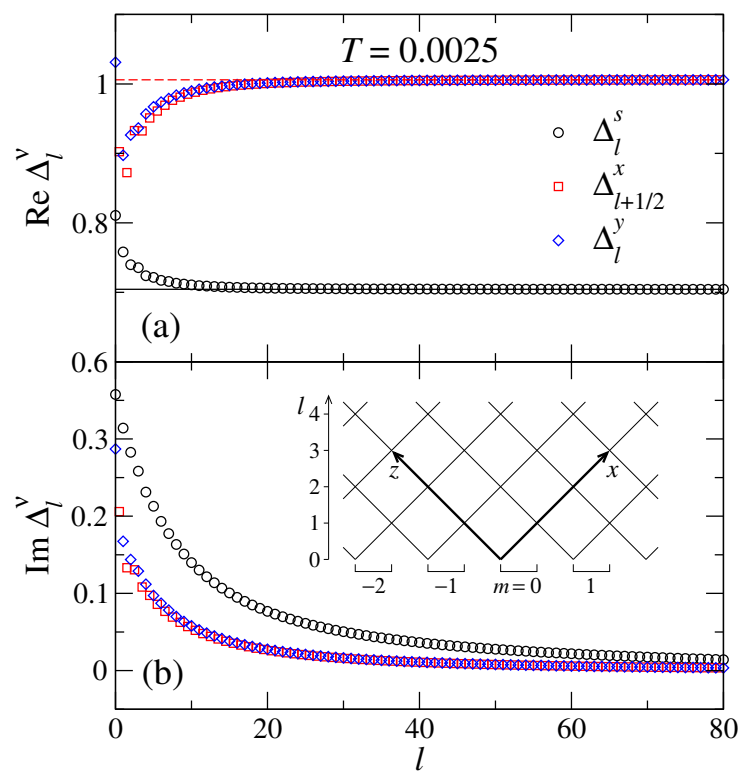

FIG. 2. (Color online) Selfconsistent gaps $\Delta_{l}^{s}, \Delta_{l+1 / 2}^{x}, \Delta_{l}^{y}$ for a slab of thickness $W=300$ and parameters as in Fig. 1 (a) and (b) show the real and imaginary parts, respectively. The lines denote the bulk gaps $\Delta_{s}$ (solid black) and $\Delta_{t}$ (dashed red). Inset: Sketch of the bottom $(l=0)$ surface of a (101) slab, showing the new coordinates $l$ and $m$. The $y$ axis points into the plane of the drawing.

$$
i U_{t}\left\langle c_{j}^{T} i \sigma^{y} \boldsymbol{\sigma} c_{i}\right\rangle \equiv \begin{cases}\Delta_{l+1 / 2}^{x} \hat{\mathbf{z}} \times \hat{\mathbf{e}}_{i j} & \text { for } x \text { bonds } \\ \Delta_{l}^{y} \hat{\mathbf{z}} \times \hat{\mathbf{e}}_{i j} & \text { for } y \text { bonds }\end{cases}
$$

where the subscript $l$ denotes the (identical) $l$ coordinate of sites $i$ and $j$, while $l+1 / 2$ in $\Delta_{l+1 / 2}^{x}$ is the mean of the $l$ coordinates of sites $i$ and $j$. We Fourier transform in the directions parallel to the slab, introducing the two-dimensional momentum vector $\mathbf{k}=\left(k_{m}, k_{y}\right)$ in the surface BZ, defined by $-\pi<k_{y} \leq \pi$ and $-\pi / \sqrt{2}<$ $k_{m} \equiv\left(k_{x}-k_{z}\right) / \sqrt{2} \leq \pi / \sqrt{2}$. The MF calculations are performed for a slab of thickness $W=300$, using the same parameters as for the bulk calculation. Further details are presented in Sec. II of the Supplemental Material 43 .

Spontaneous breaking of TRS. Our central results are summarized in Figs. 2 and 3 at sufficiently low temperatures, the singlet and triplet gaps develop imaginary components close to the surface, spontaneously breaking TRS. This solution is degenerate with a state with complex-conjugated gaps. In the limit $W \rightarrow \infty$, the two surfaces are decoupled and there are hence four degenerate TRSB solutions, differing in the signs of the imaginary parts of the gaps close to the surfaces.

The spatial variation of the gaps near the surface in the TRSB phase is shown in Fig. 2. While both the singlet and triplet gaps develop imaginary components near the surface, the real parts of the singlet and triplet gaps are enhanced above and suppressed below their bulk values, respectively. The suppression of the triplet gaps origi- 


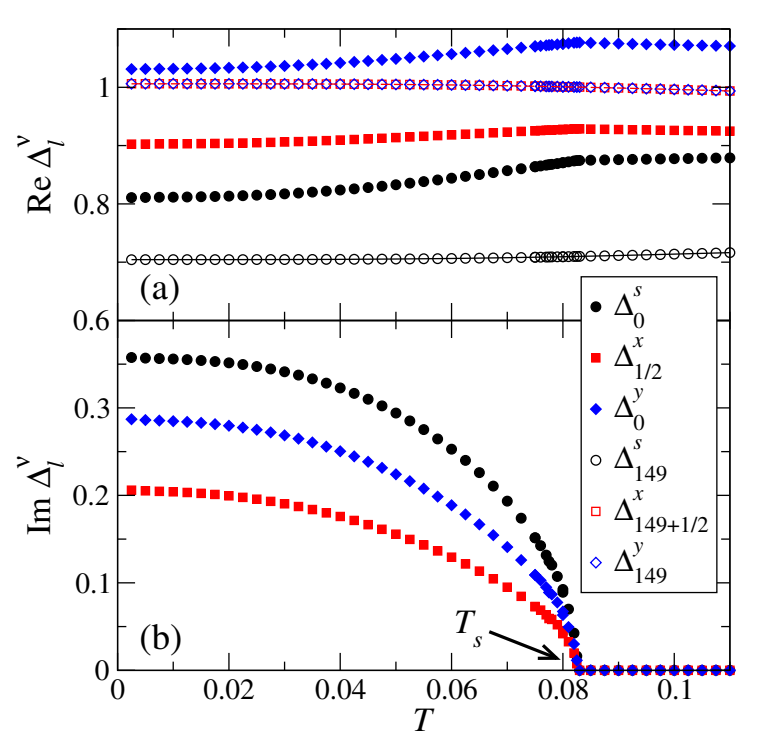

FIG. 3. (Color online) Selfconsistent gaps $\Delta_{l}^{s}, \Delta_{l+1 / 2}^{x}, \Delta_{l}^{y}$ for the surface layer ( $l=0$, filled symbols) and at the slab center ( $l=W / 2-1$, open symbols) as functions of temperature. The thickness is $W=300$, the parameters are as in Fig. 1 1 (a) and (b) show the real and imaginary parts, respectively. The imaginary parts for $l=W / 2-1$ would be indistinguishable from zero and are omitted. The lines in panel (a) denote the bulk gaps $\Delta_{s}$ (solid black) and $\Delta_{t}$ (dashed red) from Fig. 1(b).

nates from the pair-breaking effect of the surface, which in turn enhances the singlet gap to compensate for the lost condensation energy. The reversal of the suppression of the triplet gaps in the outermost layer can be understood similarly: since one of the triplet amplitudes is missing at the surface, the others are enhanced.

The gaps converge to their bulk values as we move away from the surface; the gaps at the center of the slab are within $0.01 \%$ of their bulk values. Note that the deviation of the imaginary parts from their bulk value (of zero) has a much longer range than that of the real parts. Indeed, close to the center of the slab, we find that $\operatorname{Im} \Delta_{l}^{\nu} \propto(l-W / 2)$, see Fig. 2(b). We have checked that the proportionality constant decreases more rapidly than $W^{-1 / 2}$ with $W$ so that the gradient energy vanishes for $W \rightarrow \infty$. We attribute the slow spatial decay to the enhancement of length scales close to the bulk quantum phase transition to a nodeless singlet-dominated state. This transition can be reached by increasing $U_{s}$ and decreasing $U_{t}$ by only 0.067 (not shown).

The evolution of the TRSB state with temperature is shown in Fig. 3. where we plot the gaps $\Delta_{l}^{s}, \Delta_{l+1 / 2}^{x}$, and $\Delta_{l}^{y}$ in the surface layer and at the slab center. Upon increasing the temperature, the gaps in the surface layer show a second-order transition, at which the imaginary parts vanish and TRS is restored. This occurs at a temperature of $T_{s} \approx 0.083$, well below the bulk superconducting transition temperature $T_{c} \approx 0.942$.

Dispersion and density of states. In Fig. 4(a) we plot the dispersion for a cut through the surface $\mathrm{BZ}$ at $k_{y}=0$

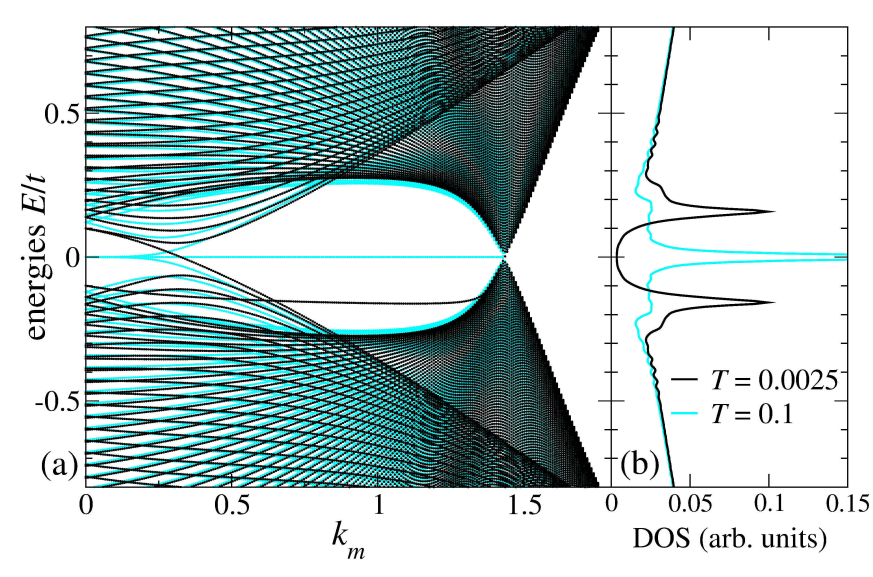

FIG. 4. (Color online) (a) Dispersion for a cut through the surface BZ at $k_{y}=0$, for $W=300$ and the same parameters as in Fig. 1. The black points refer to $T=0.0025 \ll T_{s}$ in the TRSB state, whereas the cyan (light gray) points in the background refer to $T=0.1>T_{s}$ with restored TRS. The dispersion is odd in $k_{m}$, only points for $k_{m} \geq 0$ are shown. (b) Surface DOS in the $l=0$ layer at the same temperatures. An artificial broadening of $\eta=0.01$ was used.

at temperatures below and above $T_{s}$. For $T>T_{s}$, the zero-energy flat band predicted in Refs. [2, 11] is clearly visible for $0.5 \lesssim k_{m} \lesssim 1.5$; the zero-energy states at $k_{m} \lesssim 0.5$ form an arc connecting the projections of the nodal rings [2, 11. The TRSB for $T<T_{s}$ removes the topological protection of the zero-energy flat bands of the TRS state, which are consequently pushed away from zero energy, with a low-temperature energy shift on the order of $T_{s}$. Since the shift is weakly momentum dependent, the band obtains a nonzero velocity. Due to particle-hole symmetry, the dispersion is odd in $\mathbf{k}$. The zero-energy flat bands give a singular contribution to the surface density of states, which can be detected as a sharp zero-bias peak in the tunneling spectrum of an NCS-normal-metal junction [1, 10, 11]. The shift of the surface bands in the TRSB state causes a splitting of this peak, as shown in Fig. 4(b). This splitting is a key experimental signature of TRSB. Indeed, the observed splitting of the zero-bias peak for tunneling into the (110) surface of the cuprates is important evidence for TRSB in this system [31, 32].

Spin polarization. Broken TRS is also manifested by a nonzero spin polarization near the surface, which is directed along the $y$-axis. A polarization in other directions is forbidden by mirror symmetry in the $x z$ plane. Figure 5(a) shows the spatial variation of the layer-resolved spin contributions $\left\langle s_{l}^{y}\right\rangle$; explicit expressions for the spin operator $\mathbf{s}_{l}$ in layer $l$ and its thermal average are given in Sec. III of the Supplemental Material [43. It is interesting to examine how states at different $\mathbf{k}$ contribute to the spin polarization: due to the strong polarization of the flat-band surface states in the TRS state [44, 46], one might expect that the spin polarization largely originates from the shifted flat bands. To check this, we plot 


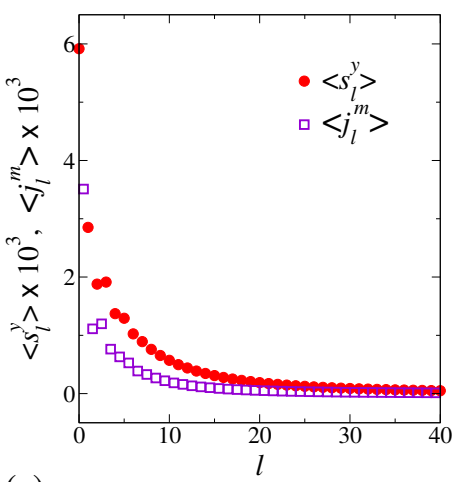

(a)

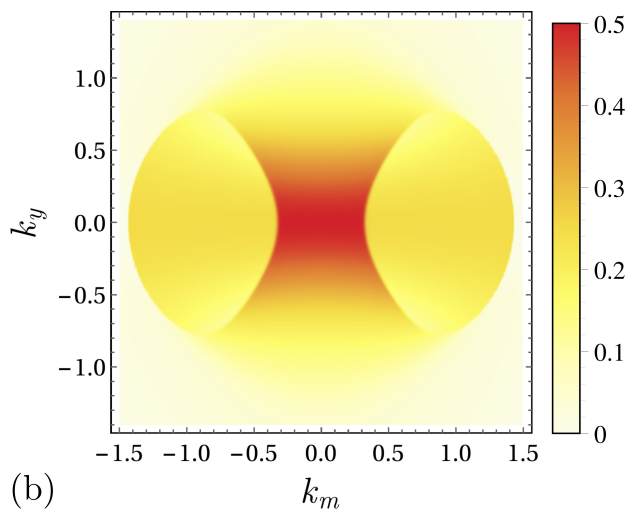

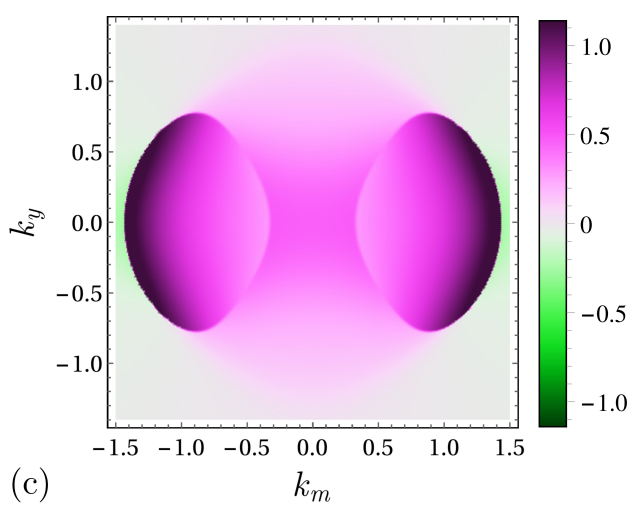

FIG. 5. (Color online) (a) spin polarization $\left\langle s_{l}^{y}\right\rangle$ (filled circles) and current $\left\langle j_{l}^{m}\right\rangle$ (open squares) as functions of $l$, for $W=300$ and the same parameters as in Fig. 1. Both quantities are given in units of their value at the surface. The layer indices of $\left\langle j_{l}^{m}\right\rangle$ are given as half integers to indicate that the current flows between two layers, see the inset of Fig. 2 (b) Momentumresolved contributions to the $y$ component of the total spin polarization of half the slab $(0 \leq l<W / 2)$ in the surface BZ. (c) Momentum-resolved contributions to the $m$ component of the current in half the slab $(0 \leq l<W / 2)$. The momentum-space plots in (b) and (c) are restricted to a region just enclosing the projection of the positive-helicity Fermi surface.

in Fig. 5(b) the momentum-resolved contribution to the spin polarization of the half slab defined by $0 \leq l<W / 2$ [43. Surprisingly, the spin polarization is not primarily carried by the shifted flat bands but rather by bulk and perhaps dispersing surface states [2, 11, from the region between the projected nodal rings.

Equilibrium currents. Furthermore, the absence of TRS permits a nonzero equilibrium surface current [22, 24, 26]. Indeed, we expect such a current since the surface bands become dispersive and the dispersion is odd in $k_{m}$; a similar modification of the electronic structure at an interface with a ferromagnet does result in a surface current [19, 20]. Explicit expressions for the current operator $\mathbf{j}_{l}$ in layer $l$ and its thermal average are given in Sec. IV of the Supplemental Material 43]. Although charge is not conserved in the superconducting MF state, one can account for the pairing potentials by adding so-called source terms to the continuity equation [47. For selfconsistently calculated gaps, however, the thermal average of the source terms vanishes, and charge conservation is retained [47. This implies that the current perpendicular to the slab's surface, i.e., in the $l$ direction, must vanish. Mirror symmetry in the $x z$ plane forbids a current along the $y$-axis 43 , leaving only the current along the $m$ direction, defined as $\left\langle j_{l+1 / 2}^{m}\right\rangle=\left(\left\langle j_{l+1 / 2}^{x}\right\rangle-\left\langle j_{l+1 / 2}^{z}\right\rangle\right) / \sqrt{2}$. $\left\langle j_{l+1 / 2}^{m}\right\rangle$ is indeed nonzero in the TRSB state: In Fig. 5 (a) we plot the current as a function of the layer index $l$, which shows that it is bound to the surface with spatial profile similar to the spin polarization. In contrast to the spin polarization, the main contribution to the current stems from surface states within the projected nodal rings, as shown by the momentum-resolved current in a half slab plotted in Fig. 5(c). We have also studied the contributions to the vanishing components $\left\langle j_{l}^{l}\right\rangle$ and $\left\langle j_{l}^{y}\right\rangle$, shown in the Supplemental Material [43. Interestingly, $\left\langle j_{l}^{l}\right\rangle$ cancels only in the sum over the full surface BZ, showing that bulk states must be included to satisfy charge conservation. Note that the sign of both the spin polarization and the current is reversed for the degenerate solution with complex-conjugated gaps.

The coupling to the electromagnetic field, which is not included here, leads to additional screening currents that exactly balance the spontaneous surface current in the limit $W \rightarrow \infty$. However, these currents build up on the length scale of the magnetic penetration depth $\lambda$, which in typical NCSs is much larger than the decay length of the surface current, on the order of the coherence length $\xi$ [12. In samples with thickness smaller than the penetration depth but larger than the coherence length, it should thus be possible to detect the surface current.

Summary and conclusions. We have studied the stability of zero-energy flat bands at the surface of an NCS within selfconsistent MF theory. We find that the flat bands are indeed recovered by the selfconsistent calculation within a broad temperature range below the bulk transition temperature $T_{c}$. TRS is spontaneously broken at a much lower temperature $T_{s}$, which is signaled by a nonuniform phase of the gaps. This destroys the topological protection for the flat bands, shifting them away from zero energy and giving them finite velocity. Figure 4 shows that at low temperatures the flat bands are displaced by an energy on the order of $T_{s}$, which is significantly smaller than the bulk gaps of order $T_{c}$. The free energy gain due to the shift of the flat bands is likely a major driver of the TRSB state, and ultimately limits $T_{s}$ as the free energy gain from the shift is reduced by the broadening of the Fermi function.

The TRSB state leads to clear experimental signatures: a splitting of the zero-bias peak in the tunneling spectrum, a nonvanishing spin polarization at the surface, and a nonvanishing equilibrium charge current parallel to the surface. The latter two effects show that the TRSB state found here is qualitatively different from that predicted for the (110) surface of cuprate supercon- 
ductors 22, 26, 30.

Acknowledgements. We thank A. P. Schnyder, R. Quieroz, and T. Neupert for helpful discussions. C. T. gratefully aknowledges support by the Deutsche Forschungsgemeinschaft through Research Training Group
GRK 1621 and Collaborative Research Center SFB 1143. S. R. was supported by the Norwegian Research Council, Grant Nos. 205591/V20 and 216700/F20. P. M. R. B. acknowledges support from Microsoft Station Q, LPSCMTC, and JQI-NSF-PFC.
[1] A. P. Schnyder and S. Ryu, Phys. Rev. B 84, 060504(R) (2011).

[2] A. P. Schnyder, P. M. R. Brydon, and C. Timm, Phys. Rev. B 85, 024522 (2012).

[3] Y. X. Zhao and Z. D. Wang, Phys. Rev. Lett. 110, 240404 (2013).

[4] S. Matsuura, P.-Y. Chang, A. P. Schnyder, and S. Ryu, New J. Phys. 15, 065001 (2013).

[5] S. A. Yang, H. Pan, and F. Zhang, Phys. Rev. Lett. 113, 046401 (2014).

[6] A. P. Schnyder and P. M. R. Brydon, arXiv:1502.03746

[7] M. Sato, Phys. Rev. B 73, 214502 (2006).

[8] B. Béri, Phys. Rev. B 81, 134515 (2010).

[9] Y. Tanaka, Y. Mizuno, T. Yokoyama, K. Yada, and M. Sato, Phys. Rev. Lett. 105, 097002 (2010).

[10] M. Sato, Y. Tanaka, K. Yada, and T. Yokoyama, Phys. Rev. B 83, 224511 (2011); K. Yada, M. Sato, Y. Tanaka, and T. Yokoyama, ibid. 83, 064505 (2011); M. Sato and S. Fujimoto, Phys. Rev. Lett. 105, 217001 (2010).

[11] P. M. R. Brydon, A. P. Schnyder, and C. Timm, Phys. Rev. B 84, 020501(R) (2011).

[12] Non-Centrosymmetric Superconductors: Introduction and Overview, edited by E. Bauer and M. Sigrist, Lecture Notes in Physics Vol. 847 (Springer, Berlin, 2012).

[13] K. Izawa, Y. Kasahara, Y. Matsuda, K. Behnia, T. Yasuda, R. Settai, and Y. Onuki, Phys. Rev. Lett. 94, 197002 (2005).

[14] H. Q. Yuan, D. F. Agterberg, N. Hayashi, P. Badica, D. Vandervelde, K. Togano, M. Sigrist, and M. B. Salamon, Phys. Rev. Lett. 97, 017006 (2006).

[15] M. Nishiyama, Y. Inada, and G.-Q. Zheng, Phys. Rev. Lett. 98, 047002 (2007)

[16] H. Mukuda, T. Fujii, T. Ohara, A. Harada, M. Yashima, Y. Kitaoka, Y. Okuda, R. Settai, and Y. Onuki, Phys. Rev. Lett. 100, 107003 (2008).

[17] I. Bonalde, R. L. Robeiro, W. Bramer-Escamilla, C. Rojas, E. Bauer, A. Prokofiev, Y. Haga, T. Yasuda, and Y. Onuki, New J. Phys. 11, 055054 (2009).

[18] G. Eguchi, D. C. Peets, M. Kriener, S. Yonezawa, G. Bao, S. Harada, Y. Inada, G.-q. Zheng, and Y. Maeno, Phys. Rev. B 87, 161203(R) (2013).

[19] P. M. R. Brydon, C. Timm, and A. P. Schnyder, New J. Phys. 15, 045019 (2013).

[20] A. P. Schnyder, C. Timm, and P. M. R. Brydon, Phys. Rev. Lett. 111, 077001 (2013).

[21] J. S. Hofmann, R. Queiroz, and A. P. Schnyder, Phys. Rev. B 88, 134505 (2013).

[22] M. Matsumoto and H. Shiba, J. Phys. Soc. Jpn. 64, 3384 (1995); 64, 4867 (1995).

[23] M. Fogelström, D. Rainer, and J. A. Sauls, Phys. Rev. Lett. 79, 281 (1997)

[24] M. Sigrist, Prog. Theor. Phys. 99, 899 (1998).

[25] J.-X. Zhu, B. Friedman, and C. S. Ting, Phys. Rev. B 59, 3353 (1999)
[26] C. Honerkamp, K. Wakabayashi, and M. Sigrist, Europhys. Lett. 50, 368 (2000).

[27] S. Kashiwaya and Y. Tanaka, Rep. Prog. Phys. 63, 1641 (2000)

[28] A. M. Black-Schaffer, D. S. Golubev, T. Bauch, F. Lombardi, and M. Fogelström, Phys. Rev. Lett. 110, 197001 (2013).

[29] Y. Tanaka and S. Kashiwaya, Phys. Rev. Lett. 74, 3451 (1995).

[30] A. C. Potter and P. Lee, Phys. Rev. Lett. 112, 117002 (2014).

[31] M. Covington, M. Aprili, E. Paraoanu, L. H. Greene, F. Xu, J. Zhu, and C. A. Mirkin, Phys. Rev. Lett. 79, 277 (1997)

[32] R. Krupke and G. Deutscher, Phys. Rev. Lett. 83, 4634 (1999)

[33] D. Gustafsson, D. Golubev, M. Fogelström, T. Claeson, S. Kubatkin, T. Bauch, and F. Lombardi, Nature Nano. 8, 25 (2013).

[34] J. Y. T. Wei, N.-C. Yeh, D. F. Garrigus, and M. Strasik, Phys. Rev. Lett. 81, 2542 (1998).

[35] A. Biswas, P. Fournier, M. M. Qazilbash, V. N. Smolyaninova, H. Balci, and R. L. Greene, Phys. Rev. Lett. 88, 207004 (2002).

[36] H. Kashiwaya, S. Kashiwaya, B. Prijamboedi, A. Sawa, I. Kurosawa, Y. Tanaka, and I. Iguchi, Phys. Rev. B 70, 094501 (2004).

[37] B. Chesca, M. Seifried, T. Dahm, N. Schopohl, D. Koelle, R. Kleiner, and A. Tsukada, Phys. Rev. B 71, 104504 (2005); B. Chesca, D. Doenitz, T. Dahm, R. P. Huebener, D. Koelle, R. Kleiner, Ariando, H. J. H. Smilde, and H. Hilgenkamp, ibid. 73, 014529 (2006); B. Chesca, H. J. H. Smilde, and H. Hilgenkamp, ibid. 77, 184510 (2008).

[38] M. Wagenknecht, D. Koelle, R. Kleiner, S. Graser, N. Schopohl, B. Chesca, A. Tsukada, S. T. B. Goennenwein, and R. Gross, Phys. Rev. Lett. 100, 227001 (2008).

[39] E. Bauer, G. Hilscher, H. Michor, C. Paul, E. W. Scheidt, A. Gribanov, Y. Seropegin, H. Noël, M. Sigrist, and P. Rogl, Phys. Rev. Lett. 92, 027003 (2004).

[40] N. Kimura, K. Ito, K. Saitoh, Y. Umeda, H. Aoki, and T. Terashima, Phys. Rev. Lett. 95, 247004 (2005).

[41] I. Sugitani, Y. Okuda, H. Shishido, T. Yamada, A. Thamizhavel, E. Yamamoto, T. D. Matsuda, Y. Haga, T. Takeuchi, R. Settai, and Y. Ōnuki, J. Phys. Soc. Jpn. 75, 043703 (2006).

[42] P. A. Frigeri, D. F. Agterberg, A. Koga, and M. Sigrist, Phys. Rev. Lett. 92, 097001 (2004).

[43] See Supplemental Material (attached) for details on the MF theory and on the calculation of the spin per site and the charge current.

[44] P. M. R. Brydon, A. P. Schnyder, and C. Timm, New J. Phys. 17, 013016 (2015).

[45] Note that the sign of the SOC is inverted compared to Refs. [2, 11, 44]. 
[46] R. Queiroz and A. P. Schnyder, Phys. Rev. B 89, 054501 (2014).
[47] A. Furusaki and M. Tsukada, Solid State Commun. 78, 299 (1991). 


\title{
Supplemental Material for Surface instability in nodal noncentrosymmetric superconductors
}

\author{
Carsten Timm, Stefan Rex, and P. M. R. Brydon
}

\section{MEAN-FIELD THEORY FOR THE BULK}

In this section we sketch the MF theory for the bulk NCS. We assume spatially uniform pairing potentials $\Delta_{j}^{s}=\Delta_{s}$ and $\Delta_{i j}^{t}=\Delta_{t}$. Using this ansatz to decouple the interaction Hamiltonian $H_{\text {int }}$, we obtain the Bogoliubov-de Gennes (BdG) Hamiltonian [S1]

$$
H_{\mathrm{MF}}=\frac{1}{2} \sum_{\mathbf{k}} \Phi_{\mathbf{k}}^{\dagger} \mathcal{H}(\mathbf{k}) \Phi_{\mathbf{k}}+N \frac{\Delta_{s}^{2}}{U_{s}}+N \frac{\Delta_{t}^{2}}{U_{t}}
$$

with the number of sites, $N$, and the block matrix

$$
\mathcal{H}(\mathbf{k})=\left(\begin{array}{cc}
h(\mathbf{k}) & \Delta(\mathbf{k}) \\
\Delta^{\dagger}(\mathbf{k}) & -h^{T}(-\mathbf{k})
\end{array}\right)
$$

written in terms of $h(\mathbf{k})=\xi_{\mathbf{k}} \sigma^{0}-\lambda \mathbf{l}_{\mathbf{k}} \cdot \boldsymbol{\sigma}, \Delta(\mathbf{k})=\left(\Delta_{s} \sigma^{0}+\Delta_{t} \mathbf{l}_{\mathbf{k}} \cdot \boldsymbol{\sigma}\right) i \sigma^{y}, \xi_{\mathbf{k}}=-2 t\left(\cos k_{x}+\cos k_{y}+\cos k_{z}\right)-\mu$, $\mathbf{l}_{\mathbf{k}}=\hat{\mathbf{x}} \sin k_{y}-\hat{\mathbf{y}} \sin k_{x}$, and the Nambu spinor $\Phi_{\mathbf{k}}=\left(c_{\mathbf{k} \uparrow}, c_{\mathbf{k} \downarrow}, c_{-\mathbf{k}, \uparrow}^{\dagger}, c_{-\mathbf{k}, \downarrow}^{\dagger}\right)^{T}$. Here, $\sigma^{0}$ is the $2 \times 2$ identity matrix. The dispersion $E_{\mathbf{k} \nu}, \nu=1, \ldots, 4$ is obtained by diagonalizing $\mathcal{H}(\mathbf{k}) . \Delta_{s}$ and $\Delta_{t}$ are then obtained by minimizing the free energy

$$
F_{\mathrm{MF}}=-k_{B} T \sum_{\mathbf{k} \nu}^{\prime} \ln \left(2 \cosh \frac{\beta E_{\mathbf{k} \nu}}{2}\right)+N \frac{\Delta_{s}^{2}}{U_{s}}+N \frac{\Delta_{t}^{2}}{U_{t}},
$$

where the momentum sum is over half the BZ, $k_{m}>0$. This restriction of the sum makes use of particle-hole symmetry, which relates the Hamiltonian in Eq. S2 at $\mathbf{k}$ and $-\mathbf{k}$ by [S1] $\mathcal{U}_{C} \mathcal{H}^{T}(-\mathbf{k}) \mathcal{U}_{C}^{\dagger}=-\mathcal{H}(\mathbf{k})$ with the unitary matrix $\mathcal{U}_{C}=\sigma^{x} \otimes \sigma^{0}$.

\section{MEAN-FIELD THEORY FOR THE SLAB}

We now set up the MF Hamiltonian for the (101) slab and describe the determination of the gap parameters $\Delta_{l}^{s}$, $\Delta_{l+1 / 2}^{x}$, and $\Delta_{l}^{y}$ in the MF approximation. After Fourier transformation in the directions parallel to the surfaces, the MF Hamiltonian reads

$$
\begin{aligned}
H_{\mathrm{MF}}= & \frac{1}{2} \sum_{\mathbf{k}} \sum_{l=0}^{W-1} \Phi_{\mathbf{k} l}^{\dagger} \mathcal{H}_{l l}(\mathbf{k}) \Phi_{\mathbf{k} l}+\frac{1}{2} \sum_{\mathbf{k}} \sum_{l=0}^{W-2} \Phi_{\mathbf{k}, l+1}^{\dagger} \mathcal{H}_{l+1, l}(\mathbf{k}) \Phi_{\mathbf{k} l}+\frac{1}{2} \sum_{\mathbf{k}} \sum_{l=1}^{W-1} \Phi_{\mathbf{k}, l-1}^{\dagger} \mathcal{H}_{l-1, l}(\mathbf{k}) \Phi_{\mathbf{k} l} \\
& +\frac{N_{\|}}{U_{s}} \sum_{l=0}^{W-1}\left|\Delta_{l}^{s}\right|^{2}+\frac{N_{\|}}{2 U_{t}} \sum_{l=0}^{W-2}\left|\Delta_{l+1 / 2}^{x}\right|^{2}+\frac{N_{\|}}{2 U_{t}} \sum_{l=0}^{W-1}\left|\Delta_{l}^{y}\right|^{2},
\end{aligned}
$$

where $N_{\|}$is the number of unit cells of the slab and $\Phi_{\mathbf{k} l}=\left(c_{\mathbf{k} l \uparrow}, c_{\mathbf{k} l \downarrow}, c_{-\mathbf{k}, l, \uparrow}^{\dagger}, c_{-\mathbf{k}, l, \downarrow}^{\dagger}\right)^{T}$ is the partially Fouriertransformed Nambu spinor. The sums over $l$ containing $\Phi_{\mathbf{k}, l \pm 1}^{\dagger}$ are restricted in such a way that $l \pm 1 \in\{0, \ldots, W-1\}$. The coefficient matrices appearing in $H_{\mathrm{MF}}$ are

$$
\begin{aligned}
\mathcal{H}_{l l}(\mathbf{k}) & =\left(\begin{array}{cccc}
-2 t \cos k_{y}-\mu & -\lambda \sin k_{y} & -\Delta_{l}^{y} \sin k_{y} & \Delta_{l}^{s} \\
-\lambda \sin k_{y} & -2 t \cos k_{y}-\mu & -\Delta_{l}^{s} & \Delta_{l}^{y} \sin k_{y} \\
-\Delta_{l}^{y *} \sin k_{y} & -\Delta_{l}^{s *} & 2 t \cos k_{y}+\mu & -\lambda \sin k_{y} \\
\Delta_{l}^{s *} & \Delta_{l}^{y *} \sin k_{y} & -\lambda \sin k_{y} & 2 t \cos k_{y}+\mu
\end{array}\right), \\
\mathcal{H}_{l \pm 1, l}(\mathbf{k}) & =\left(\begin{array}{cccc}
-2 t \cos \left(k_{m} / \sqrt{2}\right) & \pm(\lambda / 2) e^{\mp i k_{m} / \sqrt{2}} & \pm\left(\Delta_{l \pm 1 / 2}^{x} / 2\right) e^{\mp i k_{m} / \sqrt{2}} & 0 \\
\mp(\lambda / 2) e^{\mp i k_{m} / \sqrt{2}} & -2 t \cos \left(k_{m} / \sqrt{2}\right) & 0 & \pm\left(\Delta_{l \pm 1 / 2}^{x} / 2\right) e^{\mp i k_{m} / \sqrt{2}} \\
\mp\left(\Delta_{l \pm 1 / 2}^{x *} / 2\right) e^{\mp i k_{m} / \sqrt{2}} & 0 & 2 t \cos \left(k_{m} / \sqrt{2}\right) & \mp(\lambda / 2) e^{\mp i k_{m} / \sqrt{2}} \\
0 & \mp\left(\Delta_{l \pm 1 / 2}^{x *} / 2\right) e^{\mp i k_{m} / \sqrt{2}} & \pm(\lambda / 2) e^{\mp i k_{m} / \sqrt{2}} & 2 t \cos \left(k_{m} / \sqrt{2}\right)
\end{array}\right) .
\end{aligned}
$$


We next construct the $4 W \times 4 W$ block matrix

$$
\mathcal{H}(\mathbf{k}) \equiv\left(\begin{array}{cccc}
\mathcal{H}_{00}(\mathbf{k}) & \mathcal{H}_{01}(\mathbf{k}) & 0 & \ldots \\
\mathcal{H}_{10}(\mathbf{k}) & \mathcal{H}_{11}(\mathbf{k}) & \mathcal{H}_{12}(\mathbf{k}) & \ldots \\
0 & \mathcal{H}_{21}(\mathbf{k}) & \mathcal{H}_{22}(\mathbf{k}) & \cdots \\
\vdots & \vdots & \vdots & \ddots
\end{array}\right)
$$

and denote its eigenvalues by $E_{\mathbf{k} \nu}, \nu=1, \ldots, 4 W$ and the corresponding eigenvectors by $|\mathbf{k} \nu\rangle$. The MF Hamiltonian satisfies particle-hole symmetry [1], $\mathcal{U}_{C} \mathcal{H}^{T}(-\mathbf{k}) \mathcal{U}_{C}^{\dagger}=-\mathcal{H}(\mathbf{k})$ with the unitary matrix $\mathcal{U}_{C}=\mathbb{1}_{W} \otimes \sigma^{x} \otimes \sigma^{0}$, where $\mathbb{1}_{W}$ is the $W \times W$ identity matrix. This symmetry again allows to restrict the momentum sums to half the BZ. The free energy can then be written as

$$
F_{\mathrm{MF}}=-k_{B} T \sum_{\mathbf{k} \nu}^{\prime} \ln \left(2 \cosh \frac{\beta E_{\mathbf{k} \nu}}{2}\right)+\frac{N_{\|}}{U_{s}} \sum_{l=0}^{W-1}\left|\Delta_{l}^{s}\right|^{2}+\frac{N_{\|}}{2 U_{t}} \sum_{l=0}^{W-2}\left|\Delta_{l+1 / 2}^{x}\right|^{2}+\frac{N_{\|}}{2 U_{t}} \sum_{l=0}^{W-1}\left|\Delta_{l}^{y}\right|^{2},
$$

where the momentum sum is restricted to half the BZ, $k_{m}>0$. Minimization of $F_{\mathrm{MF}}$ gives the gaps $\Delta_{l}^{s}, \Delta_{l+1 / 2}^{x}$, and $\Delta_{l}^{y}$. The derivatives of $F_{\mathrm{MF}}$ with respect to the complex conjugate gaps can be calculated with the help of the Hellmann-Feynman theorem, for example

$$
\frac{\partial F_{\mathrm{MF}}}{\partial \Delta_{l}^{s *}}=-\frac{1}{2} \sum_{\mathbf{k} \nu}^{\prime} \tanh \frac{\beta E_{\mathbf{k} \nu}}{2}\left\langle\mathbf{k} \nu\left|\frac{\partial \mathcal{H}(\mathbf{k})}{\partial \Delta_{l}^{s *}}\right| \mathbf{k} \nu\right\rangle+\frac{N_{\|}}{U_{s}} \Delta_{l}^{s} .
$$

The momentum sums are performed on a $50 \times 50$ mesh, referring to the full surface BZ. Quadrupling the number of points in the mesh to $100 \times 100$ leads to changes in the MF gaps on the order of only $0.1 \%$.

Solving the resulting MF equations by iteration turns out to be prohibitively slow for the required $W$, essentially because the minimum of $F_{\mathrm{MF}}$ is very shallow in some directions in the high-dimensional space of gap parameters. On the other hand, numerical minimization making use of the explicitly known gradient is reasonably efficient. We use the Broyden-Fletcher-Goldfarb-Shanno method implemented in Numerical Recipes [S2. It requires an initial guess for the inverse Hessian. When we scan over ranges of temperatures, we use not only the converged values of the gaps but also the best approximate inverse Hessian from one step as starting values for the next, which significantly speeds up the convergence. We assume that the method has converged when no real or imaginary part of any gap parameter changes by more than (double) machine precision in the last step.

For certain parameter values, we find nonvanishing gradients of the phases of the order parameters in the $l$ direction, normal to the surfaces. Specifically, we find four metastable solutions, which are mapped onto each other by inverting the phase gradients at one or both surfaces. In the limit $W \rightarrow \infty$, the four solutions are degenerate. For finite $W$, they split into two degenerate pairs with phase gradients that are even and odd, respectively, under reflection at the center of the slab. We here choose a solution with even phase gradients since then the selfconsistent solution ensures that the phases of $\Delta_{l}^{s}, \Delta_{l+1 / 2}^{x}$, and $\Delta_{l}^{y}$ become equal at the center of the slab; equal phases of all gaps at the center are expected since the bulk MF solution has equal phases. By a global phase change we can then make the phase of all gaps zero at the center. The phases and imaginary parts of the gaps are then odd under reflection at the center. Finally, of the two remaining solutions differing in the sign of the imaginary parts of the gaps, we select the solution with $\operatorname{Im} \Delta_{0}^{s} \geq 0$ for definiteness. The other solution leads to inverted spin polarizations and currents.

\section{SPIN POLARIZATION}

Here, we present expressions for the spin polarization. The operator of the spin per site, averaged over the directions parallel to the surfaces, is

$$
\mathbf{s}_{l}=\frac{1}{N_{\|}} \sum_{\mathbf{k}} c_{\mathbf{k} l}^{\dagger} \frac{\sigma}{2} c_{\mathbf{k} l}
$$

Using particle-hole symmetry, the thermal spin average can be written as

$$
\left\langle\mathbf{s}_{l}\right\rangle=-\frac{1}{4 N_{\|}} \sum_{\mathbf{k} \nu}^{\prime} \tanh \frac{\beta E_{\mathbf{k} \nu}}{2}\left\langle\mathbf{k} \nu\left|P_{l l} \otimes\left(\begin{array}{cc}
\boldsymbol{\sigma} & 0 \\
0 & -\boldsymbol{\sigma}^{T}
\end{array}\right)\right| \mathbf{k} \nu\right\rangle,
$$

where $P_{l l^{\prime}}$ is a $W \times W$ matrix with the components $\left(P_{l l^{\prime}}\right)_{n n^{\prime}}=\delta_{l n} \delta_{l^{\prime} n^{\prime}}$. We also consider the momentum-dependent contributions to the spin polarization of the half slab defined by $0 \leq l<W / 2$. These contributions are obtained by summing $\left\langle\mathbf{s}_{l}\right\rangle$ over $l=0, \ldots, W / 2-1$ and removing the factor $1 / N_{\|}$and the momentum sum. 


\section{EQUILIBRIUM CURRENT}

The second observable of interest is the current. The operators $j_{i j}^{\alpha}$ denote the electron-number current from site $j$ to its nearest neighbor $i$ in the $\alpha=x, y, z$ direction. They can be read off from $H_{0}$ in Eq. (1) in the main text,

$$
\begin{aligned}
& j_{i j}^{x}=-i c_{i}^{\dagger}\left(\begin{array}{cc}
-t & \lambda / 2 \\
-\lambda / 2 & -t
\end{array}\right) c_{j}+i c_{j}^{\dagger}\left(\begin{array}{cc}
-t & -\lambda / 2 \\
\lambda / 2 & -t
\end{array}\right) c_{i}, \\
& j_{i j}^{y}=-i c_{i}^{\dagger}\left(\begin{array}{cc}
-t & -i \lambda / 2 \\
-i \lambda / 2 & -t
\end{array}\right) c_{j}+i c_{j}^{\dagger}\left(\begin{array}{cc}
-t & i \lambda / 2 \\
i \lambda / 2 & -t
\end{array}\right) c_{i}, \\
& j_{i j}^{z}=-i c_{i}^{\dagger}\left(\begin{array}{cc}
-t & 0 \\
0 & -t
\end{array}\right) c_{j}+i c_{j}^{\dagger}\left(\begin{array}{cc}
-t & 0 \\
0 & -t
\end{array}\right) c_{i} .
\end{aligned}
$$

The interaction term $H_{\text {int }}$ conserves charge locally and therefore does not contribute to the current operator. After the MF decoupling, the anomalous terms do not conserve charge - they describe creation or annihilation of two electrons either at the same site or at neighboring sites. Such processes do not lead to currents but do introduce a source term, which is discussed in the main text. We average the current over layers parallel to the surface, taking into account that $j_{i j}^{x}$ and $j_{i j}^{z}$ connect adjacent layers, whereas $j_{i j}^{y}$ describes a current within a single layer. We then obtain the thermal averages, again using particle-hole symmetry,

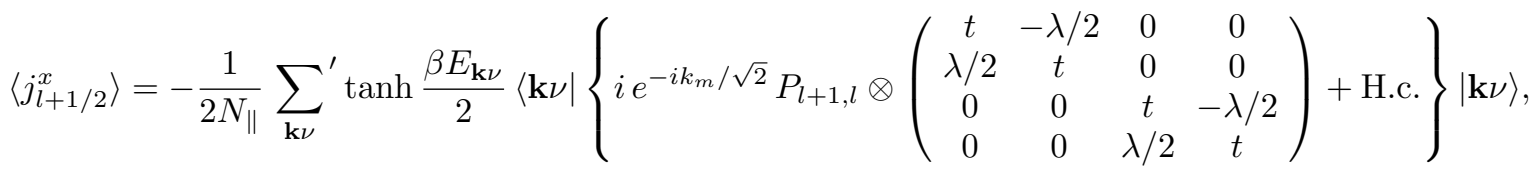

$$
\begin{aligned}
& \left\langle j_{l}^{y}\right\rangle=-\frac{1}{N_{\|}} \sum_{\mathbf{k} \nu}^{\prime} \tanh \frac{\beta E_{\mathbf{k} \nu}}{2}\left\langle\mathbf{k} \nu\left|P_{l l} \otimes\left(\begin{array}{cccc}
t \sin k_{y} & -(\lambda / 2) \cos k_{y} & 0 & 0 \\
-(\lambda / 2) \cos k_{y} & t \sin k_{y} & 0 & 0 \\
0 & 0 & t \sin k_{y} & (\lambda / 2) \cos k_{y} \\
0 & 0 & (\lambda / 2) \cos k_{y} & t \sin k_{y}
\end{array}\right)\right| \mathbf{k} \nu\right\rangle \text {, } \\
& \left\langle j_{l+1 / 2}^{z}\right\rangle=-\frac{1}{2 N_{\|}} \sum_{\mathbf{k} \nu}^{\prime} \tanh \frac{\beta E_{\mathbf{k} \nu}}{2}\langle\mathbf{k} \nu|\left\{i e^{i k_{m} / \sqrt{2}} P_{l+1, l} \otimes\left(\begin{array}{cccc}
t & 0 & 0 & 0 \\
0 & t & 0 & 0 \\
0 & 0 & t & 0 \\
0 & 0 & 0 & t
\end{array}\right)+\text { H.c. }\right\}|\mathbf{k} \nu\rangle
\end{aligned}
$$

where $\left\langle j_{l+1 / 2}^{x, z}\right\rangle$ denotes currents connecting layers $l$ and $l+1$. The components with respect to the slab coordinates are

$$
\left\langle j_{l+1 / 2}^{l}\right\rangle=\frac{\left\langle j_{l+1 / 2}^{x}\right\rangle+\left\langle j_{l+1 / 2}^{z}\right\rangle}{\sqrt{2}}, \quad\left\langle j_{l+1 / 2}^{m}\right\rangle=\frac{\left\langle j_{l+1 / 2}^{x}\right\rangle-\left\langle j_{l+1 / 2}^{z}\right\rangle}{\sqrt{2}} .
$$

We note that $\left\langle j_{l}^{y}\right\rangle$ vanishes for any choice of gap parameters for our model, even non-selfconsistent ones. This is based on mirror symmetry in the $x z$ plane. The current in the $y$ direction changes sign under this symmetry operation and thus vanishes.

The momentum-dependent contributions to the current in the half slab $0 \leq l<W / 2$ are obtained by summing $\left\langle\mathbf{j}_{l}\right\rangle$ over $l=0, \ldots, W / 2-1$ and removing the factor $1 / N_{\|}$and the momentum sum. The momentum-resolved $m$ component, which sums to a nonzero current, is shown in Fig. 5(c) in the main text. We present the momentumresolved $y$ and $l$ components in Fig. [S1. The $y$ components chancel by symmetry, as noted above. The cancelation of the $l$ components, which is required by charge conservation, is only ensured for selfconsistent gaps [S3]. Large positive contributions from bulk states within the projected (small) positive-helicity Fermi surface are canceled by small negative contributions from the flat bands and from bulk states within the projected (large) negative-helicity Fermi surface. This shows that the bulk states must be included to satisfy charge conservation.

[S1] A. P. Schnyder, P. M. R. Brydon, and C. Timm, Phys. Rev. B 85, 024522 (2012).

[S2] W. H. Press, S. A. Teukolsky, W. T. Vetterling, and B. P. Flannery, Numerical Recipes: The Art of Scientific Computing, 3rd edition (Cambridge University Press, New York, 2007).

[S3] A. Furusaki and M. Tsukada, Solid State Commun. 78, 299 (1991). 
(a)
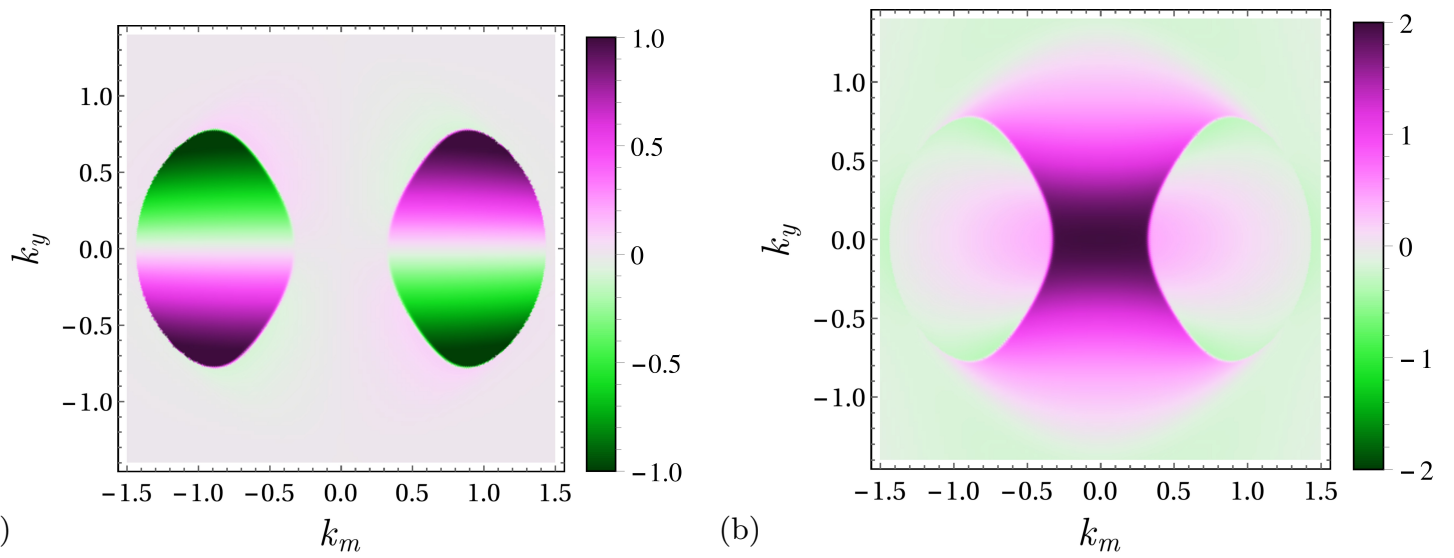

FIG. S1. Momentum-resolved contributions to (a) the $y$ component and (b) the $l$ component of the current in half the slab $(0 \leq l<W / 2)$. 